\title{
Le projet de réglementation Reach et ses liens avec la lipochimie
}

\author{
Lionel GODEFROY \\ Directeur Affaires réglementaires \\ Stepan Europe, BP 127, Chemin Jongkind, \\ 38340 Voreppe, France. \\ Fax: 0033476505135 \\ <lionel.godefroy@stepaneurope.com>
}

\begin{abstract}
This paper is showing the possible impact of the EU REACh draft regulation on the lipochemistry. The REACh regulation is quickly described. If quite a lot of oleochemicals are potentially outside the scope of REACh, (Annex 4...) and notably products used in food area, a lot of other oleochemicals will fall into its scope and will have to go the full REACh process: Registration, Evaluation and even possibly Authorisation steps for these Chemicals. An estimate is given on the coming steps for this draft regulation, particularly the second lecture step at the EU parliament that will start soon. The second lecture EU Parliament planary vote is expected at the end of this year and the publication of the final version of this REACh regulation is supposed to be at the beguinning of next year. No big modifications of this text are expected at this step, but some are still possible like the content of the Annex 4 that will not be in the scope of REACh (-previewsly Annex 2). The regulation will enter into force 20 days after its publication.
\end{abstract}

Key words: REACh, lipochemistry, impact

évaluations de risque. Ce travail va se caractériser substance par substance, par des rapports sur la sécurité des produits.

Pour un certain nombre de substances à haut risque, carcinogènes notamment, une étape ultime d'autorisation de mise sur le marché doit être effectuée au cours de laquelle doivent être définies les mesures de réduction des risques identifiés.

Tel est le processus général qui sous-tend ce projet de réglementation au service de cet objectif de sécurité pour l'homme et l'environnement.

Plusieurs objectifs ont été conférés à ce projet de réglementation. Le premier objectif visé par ce projet est le renforcement de la sécurité globale des produits chimiques, tant au niveau de l'homme qu'au niveau de l'environnement. II s'agit également à travers ce texte d'harmoniser les différentes réglementations existantes au niveau européen (par exemple, couvrir anciennes et nouvelles substances par un seul texte). Enfin, il s'agira également de mettre à jour, voire de remplacer, un certain nombre de directives européennes sur ces sujets.

Reach est un processus: Reach est un acronyme constitué à partir des termes anglais Registration, Evaluation, Autorisation, Chemicals (enregistrement, évaluation, autorisation, produits chimiques) et désignant les quatre phases de ce processus.

- L'enregistrement : cette première étape doit permettre d'établir l'inventaire des substances produites et importées.

- L'évaluation: il s'agit ici de renforcer la connaissance des substances chimiques utilisées en évaluant leur éventuelle dangerosité dont on va tirer des niveaux d'exposition selon les usages. En utilisant les données d'exposition, cette étape doit permettre d'établir les

\section{L'état du projet}

La première version de texte de ce règlement vient de la Commission européenne et a été publiée en septembre 2003, ce texte faisait au total environ 1200 pages.

Le texte a subi un premier examen au Parlement européen en novembre dernier, près de 5000 amendements ont été examinés, près de 300 amendements ont été retenus et votés en session plénière.

Le Conseil de l'Europe a alors examiné le texte du Parlement et ses délibérations ont abouti en décembre dernier à un accord politique.

Suite à cet accord, la Commission a fourni un nouveau texte "position commune " qui représentera une synthèse des trois entités (le Parlement, la Commission, et le Conseil). Ce texte qui devra être disponible dans toutes les langues européennes, a été publié en juin 2006.

Ce qui suit se base essentiellement sur cette dernière position commune du Conseil.

\section{Domaines d'application}

Dans l'état actuel du texte, quels sont les domaines d'applications?

Ce texte couvre des volumes importants puisqu'il concerne toutes les substances fabriquées à plus de $1 \mathrm{~T} /$ an par entreprise.

Il couvre les intermédiaires (partiellement), les substances importées - ces dernières seront réglementées au même titre que les substances produites - et les substances dans les articles (et également partiellement dans la mesure où elles peuvent être relarguées dans l'environnement).

Si I'on examine ce qu'il ne couvre pas, il apparaît que :

- les polymères sont pour l'instant exclus de ce projet (ils sont difficiles à décrire et ce sont en général des substances non dangereuses);

- les intermédiaires non isolés (qui restent par exemple au niveau d'un réacteur et qui sont immédiatement transformés) sont aussi exclus ;

- les intermédiaires isolés (donc passant par des cuves de stockage) seront partiellement concernés (les dossiers seront plus légers) : relèveront de ce cas également les produits de réaction transportés dans des conditions de maîtrise complète de la chaîne chez l'utilisateur final, en particulier sans contact avec l'homme et l'environnement (ce qui sera certainement difficile à démontrer).

Ne seront également pas concernés dans la mesure où ils font déjà l'objet d'une réglementation particulière :

- les substances soumises à un contrôle douanier (en transit) ;

- les substances radioactives;

- les intermédiaires non isolés;

- les déchets. 
Tableau 1. Liste des produits exemptés d'enregistrement selon l'annexe 2 du projet de règlement

\begin{tabular}{|c|c|c|}
\hline $\mathbf{N}^{\circ}$ EINECS & Nom/Groupe & $\mathrm{N}^{\circ}$ CAS \\
\hline $200-061-5$ & D-glucitol $\mathrm{C}_{6} \mathrm{H}_{14} \mathrm{O}_{6}$ & $50-70-4$ \\
\hline $200-066-2$ & acide ascorbique $\mathrm{C}_{6} \mathrm{H}_{12} \mathrm{O}_{2}$ & $50-81-7$ \\
\hline $200-075-1$ & glucose $\mathrm{C}_{6} \mathrm{H}_{12} \mathrm{O}_{6}$ & $50-99-7$ \\
\hline $200-294-2$ & L-ysine $\mathrm{C}_{6} \mathrm{H}_{14} \mathrm{~N}_{2} \mathrm{O}_{2}$ & $56-87-1$ \\
\hline $200-312-9$ & acide palmitique, pur $\mathrm{C}_{16} \mathrm{H}_{32} \mathrm{O}_{2}$ & $57-10-3$ \\
\hline $200-313-4$ & acide stéarique, pur $\mathrm{C}_{18} \mathrm{H}_{36} \mathrm{O}_{2}$ & $57-11-4$ \\
\hline 200-334-9 & saccharose, pur $\mathrm{C}_{12} \mathrm{H}_{22} \mathrm{O}_{11}$ & $57-50-1$ \\
\hline $200-405-4$ & acétate $\alpha$-tocophérile $\mathrm{C}_{31} \mathrm{H}_{52} \mathrm{O}_{3}$ & $58-95-7$ \\
\hline $200-432-1$ & DL-métionime $\mathrm{C}_{5} \mathrm{H}_{1} \mid \mathrm{NO}_{2} \mathrm{~S}$ & $59-51-8$ \\
\hline $200-711-8$ & D-mannitol $\mathrm{C}_{6} \mathrm{H}_{14} \mathrm{O}_{6}$ & $69-65-8$ \\
\hline $201-771-8$ & L-sorbose $\mathrm{C}_{6} \mathrm{H}_{12} \mathrm{O}_{6}$ & $87-79-6$ \\
\hline 204-007-1 & acide oléique, pur $\mathrm{C}_{18} \mathrm{H}_{34} \mathrm{O}_{2}$ & $112-80-1$ \\
\hline 204-664-9 & Stéarate de glycérol, pur $\mathrm{C}_{21} \mathrm{H}_{42} \mathrm{O}_{4}$ & $123-38-9$ \\
\hline $204-696-9$ & dioxyde de carbone $\mathrm{CO}_{2}$ & $124-38-9$ \\
\hline \multirow[t]{2}{*}{$205-278-9$} & pantothénate de calcium, forme $D$ & $137-08-6$ \\
\hline & $\mathrm{C}_{9} \mathrm{H}_{17} \mathrm{NO}_{512} \mathrm{Ca}$ & \\
\hline $205-782-1$ & acide laurique, pur $\mathrm{C}_{32} \mathrm{H}_{24} \mathrm{O}_{2}$ & $143-07-7$ \\
\hline $205-590-5$ & oléate de potassium $\mathrm{C}_{18} \mathrm{H}_{34} \mathrm{O}_{2} \mathrm{~K}$ & 143-18-0 \\
\hline $205-756-7$ & DL-phénylalamine $\mathrm{C}_{9} \mathrm{H}_{11} \mathrm{NO}_{2}$ & $150-30-1$ \\
\hline $208-407-7$ & gluconate de sodium $\mathrm{C}_{6} \mathrm{H}_{12} \mathrm{O}_{7} \mathrm{Na}$ & $527-07-1$ \\
\hline $212-490-5$ & stéarate de sodium, pur $\mathrm{C}_{18} \mathrm{H}_{36} \mathrm{O}_{2} \mathrm{Na}$ & $822-16-2$ \\
\hline \multirow{2}{*}{$215-279-6$} & calcaire & $1317-65-3$ \\
\hline & Solide non combustible caractéristique des roches sédimentaires. Principalement constitué de carbonate de calcium & \\
\hline $215-665-4$ & oléate de Sodium $\mathrm{C}_{24} \mathrm{H}_{44} \mathrm{O}_{6}$ & $1338-43-8$ \\
\hline $216-472-8$ & distérate de calcium, pur $\mathrm{C}_{15} \mathrm{H}_{36} \mathrm{O}_{212} \mathrm{Ca}$ & $1592-23-0$ \\
\hline $231-147-0$ & $\operatorname{argon} \mathrm{Ar}$ & 7440-37-1 \\
\hline $231-153-3$ & carbone $\mathrm{C}$ & $7440-44-0$ \\
\hline 231-783-9 & azote $\mathrm{N}_{2}$ & $7727-37-9$ \\
\hline $231-791-2$ & eaux distillées, conductibilité ou de même degré de pureté $\mathrm{H}_{2} \mathrm{O}$ & $7732-18-5$ \\
\hline $231-955-3$ & graphite C & $7782-42-5$ \\
\hline \multirow[t]{2}{*}{ 232-273-9 } & huile de tournesol & $8001-21-6$ \\
\hline & $\begin{array}{l}\text { Extraits et leurs dérivés physiquement modifiés. Se composent essentiellement de glycérides linoléique et oléique. } \\
\text { Hélianthus annuus Compositae. }\end{array}$ & \\
\hline \multirow[t]{2}{*}{$232-274-4$} & huile de soja & $8001-22-7$ \\
\hline & $\begin{array}{l}\text { Extraits et leurs dérivés physiquement modifiés. Se composent essentiellement de glycérides linoléique et oléique, } \\
\text { palmitique et stéarique (Soja hispido, Leguminosae) }\end{array}$ & \\
\hline \multirow[t]{2}{*}{$232-276-5$} & huile de carthame & $8001-23-8$ \\
\hline & $\begin{array}{l}\text { Extraits et leurs dérivés physiquement modifiés. Se composent essentiellement de glycérides de l'acide linoléique } \\
\text { (Carthhannus tinctorius, Compositae) }\end{array}$ & \\
\hline \multirow[t]{2}{*}{$232-278-6$} & huile de lin & $8001-26-1$ \\
\hline & $\begin{array}{l}\text { Extraits et leurs dérivés physiquement modifiés. Se composent essentiellement de glycérides des acides linoléique, } \\
\text { linolénique et oléique (Linum usitatissimum, Linaceae). }\end{array}$ & \\
\hline \multirow[t]{2}{*}{$232-281-2$} & huile de maïs & $8001-30-7$ \\
\hline & $\begin{array}{l}\text { Extraits et leurs dérivés physiquement modifiés. Se composent essentiellement de glycérides linoléique et oléique, } \\
\text { palmitique et stéarique (Zea mayz Gramineae) }\end{array}$ & \\
\hline \multirow[t]{2}{*}{ 232-293-8 } & huile de ricin & $8001-79-4$ \\
\hline & $\begin{array}{l}\text { Extraits et leurs dérivés physiquement modifiés. Se composent essentiellement de glycérides de l'acide ricinoléique } \\
\text { (Ricinus communis, Euphorbiacea). }\end{array}$ & \\
\hline
\end{tabular}

Par ailleurs ce règlement considère comme déjà enregistrées :

- les substances actives et co-formulants utilisés dans les produits phytopharmaceutiques et pour cet usage uniquement (Annexe 1 Dir. $91 / 414 /$ CEE) : pour un autre usage, devront se soumettre à la procédure Reach ;
- les substances actives utilisées dans les produits biocides et pour cet usage uniquement (Annexe 1... Dir. 98/8/CE).

De même les dispositions des titres II, V, VI, VII (enregistrement des substances - utilisateurs en aval évaluation - autorisation) ne seront pas applicables dans la mesure où une substance est utilisée dans :
- les médicaments à usage humain et vétérinaire (Règ. 2309/93, Dir. 2001/82,2001/83/CE) ;

- les denrées alimentaires et les aliments pour animaux (y compris comme additifs, comme substances aromatisantes) (Règ. 178/2002, Dir. 89/107/CEE, Dir. 88/388/CEE, Déc. 1999/ 217/CE, Règ. 1831/2003, Dir. 82/471/CEE). 
Tableau 2. Liste des produits exemptés d'enregistrement selon l'annexe 2 du projet de règlement

\begin{tabular}{|c|c|c|}
\hline $\mathbf{N}^{\circ}$ EINECS & Nom/Groupe & $\mathbf{N}^{\circ}$ CAS \\
\hline $232-299-0$ & $\begin{array}{l}\text { huile de colza } \\
\text { Extraits et leurs dérivés physiquement modifiés. Se composent essentiellement de glycérides des acides linoléique, } \\
\text { linolénique et oléique (brassica napus, cruciferae) }\end{array}$ & $8002-13-9$ \\
\hline $232-436-4$ & $\begin{array}{l}\text { lécithines } \\
\text { Combinaison complexe de diglycérides d'acides gras liés à l'esther formé par la choline et l'acide phosphorique. }\end{array}$ & $8002-43-5$ \\
\hline $232-436-4$ & $\begin{array}{l}\text { sirop d'amidon hydrolysé } \\
\text { Combinaison complexe obtenue par hydrolyse acide ou enzymatique d'amidon de maïs. Se composent essentiellement } \\
\text { de D-glucose, de maltose et de maltodextrines. }\end{array}$ & $8029-43-4$ \\
\hline $232-442-7$ & suif hydrogéné & $8030-12-4$ \\
\hline $232-675-4$ & Dextrine & $9005-53-9$ \\
\hline 232-679-6 & $\begin{array}{l}\text { amidon } \\
\text { Substance glucidique composée de hauts polymères généralement dérivée de graines de céréales, telles que le maïs, le } \\
\text { blé ou le sorgho, ou de racines et de tubercules, telles que le tapioca et les pommes de terres. Désigne également } \\
\text { l'amidon prégélatinisé par chauffage en présence d'eau. }\end{array}$ & $9005-25-8$ \\
\hline $232-940-4$ & Maltodextrine & $905-36-6$ \\
\hline $234-328-2$ & vitamine $\mathrm{A}$ & $11103-57-4$ \\
\hline $238-976-7$ & D-gluconate de sodium $\mathrm{C}_{6} \mathrm{H}_{12} \mathrm{O}_{7} \times \mathrm{Na}$ & 14906-97-9 \\
\hline $248-027-9$ & monostéarate de D-glucitol $\mathrm{C}_{24} \mathrm{H}_{48} \mathrm{O}_{7}$ & $26836-47-5$ \\
\hline $262-988-1$ & acides gras de coco, esters de méthyle & $61788-59-8$ \\
\hline 262-989-7 & acides gras de suif, esters de méthyle & $61788-61-2$ \\
\hline $263-060-9$ & acides gras d'huile de ricin & $61789-44-4$ \\
\hline 263-129-3 & acides gras de suif & $61790-37-2$ \\
\hline $265-995-8$ & pate de cellulose & $65996-61-4$ \\
\hline $266-925-9$ & $\begin{array}{l}\text { acides gras en } C_{12-18} \\
\text { Cette substance est répertoriée sous le SDA. Substance Name: } C_{12}-C_{18} \text { alkyl carboxylic acid et porte le SDA Reporting } \\
\text { Number } 16-005-00 \text {. }\end{array}$ & $67701-01-3$ \\
\hline $266-928-5$ & $\begin{array}{l}\text { acides gras en } C_{16-18} \\
\text { Cette substance est répertoriée sous le SDA. Substance Name: } C_{16}-C_{18} \text { alkyl carboxylic acid et porte le SDA Reporting } \\
\text { Number } 19-005-00 \text {. }\end{array}$ & $67701-03-5$ \\
\hline $266-929-0$ & $\begin{array}{l}\text { acides gras, } C_{8-18} \text { et insaturés en } C_{18} \\
\text { Cette substance est répertoriée sous le SDA. Substance Name: } C_{8}-C_{18} \text { and } C_{18} \text { unsaturated alkyl carboxylic acid et porte le } \\
\text { SDA Reporting Number } 01-005-00 \text {. }\end{array}$ & $67701-05-7$ \\
\hline $266-930-6$ & $\begin{array}{l}\text { acides gras en } C_{14-18} \text { et instaurés en } C_{16-18} \\
\text { Cette substance est répertoriée sous le SDA. Substance Name: } C_{14}-C_{18} \text { and } C_{14}-C_{18} \text { unsaturated alkyl carboxylic acid et } \\
\text { porte le SDA Reporting Number } 04-005-00 \text {. }\end{array}$ & $67701-06-8$ \\
\hline $266-932-7$ & $\begin{array}{l}\text { acides gras en } C_{14}-C_{18} \text { et instaurée en } C_{18} \\
\text { Cette substance est répertoriée sous le SDA. Substance Name: } C_{14}-C_{18} \text { and } C_{18} \text { unsaturated alkyl carboxylic acid et porte le } \\
\text { SDA Reporting Number 11-005-00. }\end{array}$ & $67701-08-0$ \\
\hline $266-948-4$ & $\begin{array}{l}\text { glycérides, } C_{16-18} \text { et instaurée en } C_{18} \\
\text { Cette substance est répertoriée sous le SDA. Substance Name: } C_{16} C_{18} \text { and } C_{18} \text { unsaturated alkyl carboxylic acid et porte le } \\
\text { SDA Reporting Number 11-001-00. }\end{array}$ & $67701-30-8$ \\
\hline $267-007-0$ & $\begin{array}{l}\text { acides gras en } C_{14}-C_{18} \text { et instaurée en } C_{14}-{ }_{18} \text { esthers de méthyle } \\
\text { Cette substance est répertoriée sous le SDA. Substance Name: } C_{14}-C_{18} \text { and } C_{14} C_{18} \text { unsaturated alkyl esther et porte le SDA } \\
\text { Reporting Number } 04-010-00 \text {. }\end{array}$ & $67762-26-9$ \\
\hline $267-013-3$ & $\begin{array}{l}\text { acides gras en } \mathrm{C}_{6-12} \\
\text { Cette substance est répertoriée sous le SDA. Substance Name: } \mathrm{C}_{6}-\mathrm{C}_{12} \text { alkyl carboxylic acid et porte le SDA Reporting } \\
\text { Number } 13-005-00 \text {. }\end{array}$ & $67762-36-1$ \\
\hline
\end{tabular}

Les dispositions des titres IV (information à l'intérieur de la chaîne d'approvisionnement) ne seront pas applicables aux préparations ci-après à l'état de produit fini, destiné à l'utilisateur final :

- les médicaments à usage humain et vétérinaire (Règ. 2309/93, Dir. 2001/82 \& 83/CE) ; - les produits cosmétiques (Dir. 76/768/CEE) ; - les dispositifs évasifs (sous certaines conditions) ;
- les denrées alimentaires et les aliments pour animaux (y compris comme additifs, comme substances aromatisantes).

\section{Domaines d'application et lipochimie}

Les produits de la lipochimie ne sont donc pas tous concernés par Reach dès lors qu'ils sont utilisés dans I'alimentation (les ingrédients et les additifs alimentaires).
II n'en reste pas moins que la lipochimie ne se limite pas à ces substances et nombre d'autres substances issues directement ou indirectement de la lipochimie entreront eux bien dans le cadre de ce règlement.

Pour ces cas-là, la charge induite pour les entreprises concernées, comportera :

- la préparation des dossiers d'enregistrement; - la constitution d'un dossier d'évaluation impliquant la génération de données (toxico- 
Tableau 3. Liste des produits exemptés d'enregistrement selon l'annexe 2 du projet de règlement

\begin{tabular}{|c|c|c|}
\hline $\mathbf{N}^{\circ}$ EINECS & Nom/Groupe & $\mathbf{N}^{\circ}$ CAS \\
\hline 268-099-5 & $\begin{array}{l}\text { acides gras en } C_{14-22} \text { et instaurés en } C_{16-22} \\
\text { Cette substance est répertoriée sous le SDA. Substance Name: } C_{14}-C_{22} \text { and } C_{16}-C_{22} \text { alkyl carboxylic acid et porte le SDA } \\
\text { Reporting Number } 07-005-00 \text {. }\end{array}$ & 68002-86-7 \\
\hline 268-616-4 & sirop de maïs déshydratés & 68131 \\
\hline $269-657-0$ & acides gras de soja & \\
\hline $269-658-6$ & glycérides de suif, mono-, di- et tri-hydrogènés & \\
\hline 270-298-7 & acide gras en $\mathrm{C}_{14-22}$ & \\
\hline 270-304-8 & acides gras d'huile de lin & \\
\hline $270-312-1$ & $\begin{array}{l}\text { glycérides en } C_{16-18} \text { et instaurés en } C_{18} \text { mono-et di } \\
\text { Cette substance est répertoriée sous le SDA. Substance Name: } C_{16}-C_{18} \text { and } C_{18} \text { unsaturated alkyl and } C_{16}-C_{18} \text { and } C_{18} \\
\text { unsaturated dialkyl glyceride et porte le SDA Reporting Number } 11-002-00 \text {. }\end{array}$ & \\
\hline 288-123-8 & glycérides en $\mathrm{C}_{10-\mathrm{C} 18}$ & $85665-33-4$ \\
\hline 292-771-7 & acides gras en $\mathrm{C}_{12-14}$ & $90990-10-6$ \\
\hline $292-776-4$ & acides gras, $C_{12-18}$ et instaurés en $C_{18}$ & $90990-10-6$ \\
\hline $296-916-5$ & acide gras d'huile de colza à basse teneur en acide érucique & $93165-15-2$ \\
\hline
\end{tabular}

logiques, physico-chimiques), de données d'exposition (d'usage), dévaluation du risque.

\section{L'annexe 4}

L'Article 2.7.a de ce projet de règlement, précise que sont exemptées des dispositions des titres II, V et VI (c'est-à-dire des procédures d'enregistrement) : « les substances figurant à l'annexe 4, si l'on dispose d'informations suffisantes à leur sujet permettant de considérer qu'elles présentent un risque minimal du fait de leurs propriétés intrinsèques».

L'annexe 4 du présent règlement, qui liste les produits exemptés d'enregistrement, se révèle donc particulièrement importante dans la mesure où figurent sur cette liste nombre de produits de la lipochimie (tableaux 1-3).

On peut mentionner notamment:

- les acides gras et certains de leurs sels ;

- les huiles (soja, colza, tournesol...) ;

- les esters type méthylique des acides gras.

Cette liste résulte d'un important travail de concertation (et de lobbying) au niveau des différentes instances de l'Union.

Elle est d'ailleurs encore susceptible d'évolution.

\section{Le devenir du projet}

La Commission européenne et le Conseil ont repris le projet pour en faire une nouvelle version pour tenir compte de l'avis du Parlement européen et de celui du Conseil. Ce projet ne diffère pas beaucoup de celui du Conseil de décembre 2005 qui lui-même est proche de celui du Parlement européen de novembre 2005.

Après la prochaine publication de cette nouvelle version du règlement par la Commission européenne, prévue pour mai/juin prochain, le nouveau texte repassera alors en deuxième lecture au Parlement européen (fin 2006-début 2007).

À ce stade, le processus devrait s'accélérer.

Selon les nouvelles modifications apportées par le Parlement, une étape dite de conciliation est à envisager pour trouver un compromis entre les positions du Parlement, du Conseil et de la Commission.

Si accord il y a, le texte final devrait donc pouvoir être publié au JOCE en 2007 ou début 2008. Dans le cas contraire, par exemple, si le
Parlement apporte des modifications trop importantes, le texte repartira à zéro.

En fait, il ne devrait pas y avoir de changements importants même si cela reste théoriquement possible, notamment au niveau des exemptions et des annexes et donc l'adoption finale de ce réglement est attendue dans le $1^{\mathrm{er}}$ semestre 2007.

En effet, théoriquement, par exemple, le domaine d'application pourrait être totalement revu (suite à certains nouveaux évènements extérieurs), comme l'ajout des polymères, ou aussi l'intégration d'autres produits présentement exclus.

Mais pour une partie au moins de ces changements, il faudrait une majorité qualifiée au Parlement européen, ce qui est loin d'être facile à obtenir.

En fait, une profonde modification du texte risquerait de le mener vers une étape de conciliation insoluble mettant ainsi en danger le texte lui-même.

Ce dernier scénario est donc peu probable politiquement même vis-à-vis des partis verts qui préféreront voir un texte imparfait s'appliquer assez vite que pas de texte du tout. 